\title{
Alimentación de recién nacidos de muy bajo peso con leche de su propia madre
}

\author{
Dra, Maria Isabal Hodgson B. ${ }^{1}$; Nutr. Bernardita Rath H.2 ;. Li. Maria Angélica Brunet B. ${ }^{3}$; \\ Dr. Ricardo Uauy D. ${ }^{3}$ : Dr. Jorge Torres P. ${ }^{1}$
}

\section{Own mother's milk for very. low birth weight newborns}

Growth was compared among ten newborn infants with birth waights less than $1500 \mathrm{~g}$ led with their own mother's milk (MM), mean birth weight $1313 \pm 128 \mathrm{~g}$, average gestational age $31.1 \pm 1.5$ weeks, vs 3 control group of identical size whost mean birth weight was $1308 \pm 166 \mathrm{~g}$, average gistational age $30.5 \pm 1.8$ week, and were ficd with an artificial formula (NAN $13 \%$. The initial weight loss was $10.9 \%$ for MM group and $9.8 \%$ for controls $(p=N, S$.), but recovery of birth weight was attained at 16.0 and 20.8 day respectively $(p<0.05)$. Subseuuent ponderal daily incroncuts were 23.7 varsus $17.5 \mathrm{~g} x$ day $(\mathrm{p}<0.005$ ). The time taken to roach $2000 \mathrm{~g}$ were $46 \pm 10$ day s in MM group and $63 \pm 17$ in tle controls $(\mathrm{p}<0.01)$. Thus own mother's milk promotes greater speed of weight gain in very low birth weight newborns than commonly used at tificial formulac.

(Key words: very low birth weight infants, growth mother's milk, artiticial formulae).

La leche materna es el alimento más adecuado para el recién nacido de término y en la actualidad su empleo como única fucnte nutriente en los primeros meses de vida es recomendado como una conducta deseable. ${ }^{1}$

Sin embargo la alimentación del recién nacido de pretérmino con leche humana es motivo de controversia. ${ }^{2-4}$ Las primeras experiencias con leche de banco (depósito de leche materna) demostraron que esta es incapaz de promover una velocidad de crecimiento similar a la observada durante el tercer trimestre de vida intrauterina $y$ que dicho crecimiento era mejor con fórmulas artificiales derivadas de leche de vaca. ${ }^{5 \cdot 7}$

Todas esas experiencias fueron hechas con leche de banco, cuyas donantes eran principalmente madres que habían tenido niños de término $y$ se encontraban en diferentes etapas de lactancia.

1. Departamento de pediatría. Pontitícia Universidad Ca tólica de Chile.

2. Instituto de Nutrición y Tecrología de los Alimentos, Univer sidad de Chlle.

3. Unidad de recién nacidos. Hospital Sóte-o del Río.

* Trabajo de ingreso a la Sociedad Chüen de Pediatría.
En los últimos años, varios estudios han demostrado que la composición de la leche humana es diferente segúl la edad gestacional en la que se produce el parto. ${ }^{8-10}$ Es asi como la leche producida por madres de niños de pretérmino, durante el primer mes de lactancia, tiene mayor contenido de proteínas y nitrógeno no protei$\mathrm{co}^{8,9,11}$ sodio, potasio y clor $\mathrm{o}^{8,12}$ que la leche de madres de recién nacidos de término, en el mismo período.

Lo anterior permite postular que la leche de la propia madre pudiese ser la fuente alimentaria más adecuada para los recién nacidos de pretér. mino, especialmente para los de muy bajo peso de nacimiento.

Este trabajo fue realizado con el propósito de comparar el crecimiento de recién nacidos de muy bajo peso, alimentados con leche de su propia madre, con un grupo similar que recibió una fórmula láctea modificada de uso frecuente en las unidades de recién nacidos.

\section{MATERIAL Y METODOS}

Se estudiaron 20 recién nacidos, seleccionados entre los que ingresaron a la unidad de recién nacidos del Hospital Sótero del Rio entre los meses de marzo de 1980 y septiembre de 1981, que cumplian con los siguientes requisitos: peso de nacimiento menor de 
$1.500 \mathrm{~g}$; adecuados para la edad gestacional; sin malfor maciones congénitas: que estuvieran en condiciones de iniciur alimentación enteral dentro de las primeras 72 horas de vida.

Su edad gestacional fue estimada por el método do Dubowitz y Dubowitz 13 y, la adecuación del peso para la edad gestacional, de acuerdo a curvas de crecimicnto intrauterino de uso internacional. 14

Las madres de los nir̃os seleccionados fueron contactadas por uno de los invistigadores (M.A.B.) para expli. carles el protocolo y obtentr su consentimiento para la extracción de leche: cuando esto no fue posible cn forma precos o existia algún impadimento. fueron asignados al grupo control. Du tita manera 10 niños formaron el grupo estudio (LM) y otros 10 el grupo control.

La leche milterna tue obtenida por extracción manuct diaria, después de aseo cuidadoso de toda la mama con agua y jabón. La r $\mathrm{r}^{2}$ colección se hizo en mamaderas estcrilizadas. desechando :n cada extracción los prime ros 2 a 3 cenrímetros cúbicos de leche. Cada mamadera fue rotulada con al nombre del nuiso, la fecha y la hora de extracción, $y$ luego almucenada a $4^{\circ}$ Celcius. La leche fuc administradit al niño dentro de las 48 horas sigujen. tes a la extracuión, a tempesatura ambjente, sin calen tamjento previo. Cuando la madie dominaba la técnica de recolección de leche y las condiciones del hogar lo perinitian (agua potable, refrigerador), se le entregaba diariamente una caja lérmica to: un vemento reftigerante y las mamaderas esterilicadas para clectuar extracción varías veces al dia. La lecle obtenidia era mantenida refrigirada a $4^{\circ} \mathrm{C}$ en el inger 3 permanecía a esta temperatura durante el transporte al hospital. Se efectuan cortroles bacteriológicos esporádicos, en forma aleatoria. para asegurar la aptifud de consumo de la leche.

Se usaron dos dietas, una constituda por la leche de la propia madre $(\mathrm{LM})$ y la atra por lecthe modificada (Nan $\left(\mathrm{E}^{2}\right.$, diluida al $13 \%$ (control). Jil volumen de leclie a administrar se planeó a $180 \mathrm{ml} \times \mathrm{kg}$ y día que se debía alcanzar a fines de la seyunda semana de vida, para luego mantener aportes entre 180 y $200 \mathrm{ml} \times \mathrm{kg} x$ diat hasta el lual del período de cstudio, considerando como tal el momento en que ol peso del niño era de $2.000 \mathrm{~g}$

Ia almentación fue hecha al inicio en forma manual intermitente por sonda nasogástrica cada 2 ó 3 horas, cambiándola posteriormente a administración por gravodad o chupete dependiendo de la madurez del reflejo de succión-deglución del nítio.

Los" requerjuicentos hidroclectrulíticos fueron comppletados con soluciones parenteralis do glucosa y electrolitos rjientras fue necesario. Todos los niños recibieron suplementos diarios de 600 v de yitamina D, $33 \mathrm{mg}$ de vitarnina C, $1.500 \mathrm{U}$ de vitamina A y 50 L.I. de vitamina $E$, desde el sciptinto dia de vida.

I a mantención de un ambiente térmico neutral y el tratamiento de las enfermedades intercurrentes fuer on efectuados de acuerdo con las nomas de la unidad de recién nacidos.

Fil peso corporal tue medido en el momento de nacer y luego diariamente utilizando la balan $\iota$ a Seca $(\mathrm{B}$ para lactantes, con precisión de \pm 10 , o pesa Air-Shields $(\mathbb{R}$ incluida en las incubadoras. Dentro de las primeras 48 horas de vida fueron también medidos la talia (cm), los perímetros craneano y braquíl derecho (mm), con huincha angosta inextensible $y$ los pliegues tricipital y subescapular (mm) mediante un calibrador lange( Todas estas mediciones fueron repetidas semanalmente hasta finalizar el período de estudio. Las mediciones de pliegue tricipal y perímetro braquial fueron utilizadas
Tabla 1.

Caracteristicas al nacer on un grupa de recién nacidus alimentados $w n$ leche de su propia madre $y$ su grupo cont rol $(x \pm$ D.E.)

\begin{tabular}{|c|c|c|}
\hline & $\begin{array}{c}\text { I.celo ina } 1 \mathrm{ct} \text { na } \\
\mathrm{n}=10\end{array}$ & $\begin{array}{c}\text { Control } \\
t=y \emptyset\end{array}$ \\
\hline Peso de nacuraituto (g! & $1313.0 \pm 128.6$ & (. $3 \mathrm{ij)} 8,0 \pm 166,0$ \\
\hline lidad gestacional (semanas) & $31,1 \pm 1,5$ & $30,5 \pm \quad 1,3$ \\
\hline Talla $( \pm n\})$ & $99,7 \pm$ & $i 3, y \pm$ \\
\hline 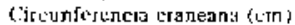 & $27,0 \pm$ & $2 ? .5 \pm$ \\
\hline Purimelro bratjujal (mIn) & $66,0 \pm$ & 65.01 \\
\hline$P \mathbf{r}+P . S \mathrm{I} .(\min )$ & $4,4 \pm \quad 0,6$ & $4,1 \pm 0,5$ \\
\hline Arca magri brdquial (mori ${ }^{2}$, & $280,0 \pm 33.0$ & $251,5 \pm 54,6$ \\
\hline Sixn $(M / 1)$ & $4: 6$ & $6 / 4$ \\
\hline
\end{tabular}

para calcular el árca magra bracuial, 15

En todos los niños se midieron homatocritos al nacer y semanaimente (microhematucrito). El porcentaje do reticulocitos y las características de los glóbulos rojos en el trotis sangu íneo tuer on determinados a $\operatorname{los} 14,28,35$ y 49 días de vida $y$, los ásus de sangre capilar en los días $14,18,22$ y 28 .

los tesultados se expresan como promedios y desvidcioncs estándar $(\bar{X} \pm D I)$. I as diferencias obsurvadas entre los grupos se comparan inediante la prueba $\{$ de Student.

\section{RESULTADOS}

Las características generales de los grupos (ML y Control) fuecon comparables al igual due los parámetros antropométricos al ingresar en el estudio (tabla l).

La calificación de Apgar a los cinco minutos fue ligeramettc superior en el grupo con leche materna $(8,4 \pm 1,0)$ que en el control $(6,8 \pm 2,0$; $p<0,05)$, pero la evolución clínica posterior de los recién nacidos fue similar en ambos. Dos niños en cadla grupo prescntaron sindrome de dificultad respiratoria y uno de cada uno recuuirió ventilación asistida en los primeros días de vida. La incidencia do hiperbilirrubinemia, septicemia $y$ ductus permeable en los primeros dias fue comparable.

Todos los recién nacidos iniciaron alimentación enteral dentro de las primeras 72 horas de vida, con volúmenes que fueron aumentados en forma gradual y progresivit, alcanzando en promedio $133 \pm 57 \mathrm{ml} \times \mathrm{kg}$ de peso en el grupo $\mathrm{LM}$ y $154 \pm 29 \mathrm{ml} \times \mathrm{kg}$ en el control a fines de la primera semana y $185 \pm 22$ vs $194 \pm 28 \mathrm{ml} \times \mathrm{kg}$ a los catorce días de vjda, respectivamente. Las diferencias ubservadas no fueron significativas.

Là tolerancia a la alimentación, evaluada clínicamente por medición de residuo gástrico previo a cada alimentación y observación de distención abdominal, fue adecuada por ambos regímenes. Dos niños en cada grupo presentaron un episodio de diarrea aguda de corta evolución, que se manejó con suspensión de los alimentos durante 24 horas seguida por realimentación progresiva Én los niños del grupo LM esto ocurrió en una 
pareja de gemelos coincidiendo con el reemplazo de leche materna por fómula láctea, un dia en que la madre no concurrió da hospital para extracción de leche. No se registraron casos de enterocolitis necrotizante en los niños estudia. dos.

La admintistración de soluciones parenterales pari cubrir requerimientos hidroelectrolíticos fuc mecesaria durante 4,4 $\pm 3,5$ días en el grupo con leche materna $y 5,3 \pm 4,6$ días en el grupo control $(p=$ N.S. $)$.

La baja de peso inicial fue similar en ambos grupos. pero la ganancia ponderal diaria desde la recuperación del peso de nacimiento lasta alcanZar los $2.000 \mathrm{~g}$ fue significativamente mayor en el grupo que recibió leche de su propia madre. El período transcurrido desde el nacimiento hasta los $2.000 \mathrm{~g} \mathrm{de}$ peso fue significativamente mayor en el grupo control (tabla 2).

Los incrementos semanales de talla, circunferencia craneana, perimetro braquial, sumatoria de pliegues tricipital $y$ subescapular (P.T. \pm P.S.E.) y área magta braquial fueron mayores en el grupo de niños que recibió leche materna, pero sólo alcanzó significación estadística para los dos úl timos parámetros mencionados (tabla 3 ).

La edad gestacional en el mumento del alta fue di $37,7 \pm 1,3$ semanas en el grupo L.M. y de $39,5 \pm 2,8$ en el control $(p<0,05)$.
En la figura l se presenta la curva de peso de ambos grupos. Los valores observados son mayo. res en el grupo L.M. a contar del día 21, pero sólo alcanzan significación estadística en el día 35 (L.M. $1.719 \pm 211 \mathrm{~g}$. control $1.526 \pm 204 \mathrm{~g}$; $\mathrm{p}<0,05)$. Algo similar se observa en la curva de talla (figura 2) donde tambièn se alcanza diferencia significativa al día 35 (L.M. 43,7 $\pm 1,7 \mathrm{~cm}$., control $41,7 \pm 22 \mathrm{~cm}$.; $p<0,025)$. En las curvas del grupo L.M. no se incluyen datos a partir del día 42, ya que 2 niños de este grupo fucron dados de alta previamente, porque habian alcanzado el objetivo de Jlegar a $2.000 \mathrm{~g}$. de peso.

E] hematocrito al nacer fue similar en anbos grupos $(53 \pm 7 \%$ en ol grupo L.M. y $52 \pm 6 \%$ en el contral), observándose una declinación progresiva y comparable al aumentar la edad posnatal. La incidencia de acidosis metabólica tardía, considerando como tal un déficit de base superior a 8 , fue igus) en ambos grupos (4 niños con L.M. y 4 controles) y los valores promedio de $\mathrm{pH}$ fueron comparables, el déficit de base fuc menor en el grupo ton leche matern's en todos los días estudiados. perola diferencia con el grupo control no fue significativa.

\section{DISCUSION}

Estos resultados demuestran que los nifios de

Tabla 2.

Lvolucion ponderal de un grupo de recién nacidos de muy bajo piso alimentados con leche de su propia madre $y$ sus controles $(\bar{x} \pm D$.E.

\begin{tabular}{|c|c|c|c|c|}
\hline Grupo & $\begin{array}{c}\text { Baja de peso } \\
\text { ( } \% \text { ) }\end{array}$ & $\begin{array}{l}\text { Recuperación } \\
\text { peso nacim. } \\
\text { (días) }\end{array}$ & $\begin{array}{l}\text { Ganancia peso } \\
\text { recup. } \mathrm{PN}->2.000 \mathrm{~g} \\
(\mathrm{~g} \times \mathrm{dia})\end{array}$ & $\begin{array}{l}\text { Tiempo desde naci- } \\
\text { miento a } 2.000 \mathrm{~g} \\
\text { (días) }\end{array}$ \\
\hline L.M. & $10.9 \pm 5,7$ & $16,0 \pm 6,1^{0}$ & $23,7 \pm 4,6^{*}$ & $46,0 \pm 10,1^{t}$ \\
\hline Control & $9.8 \pm 3.7$ & $20,8 \pm 4,3$ & $17,5 \pm 4,4$ & $63,5 \pm 17,0$ \\
\hline
\end{tabular}

\footnotetext{
$\circ \mathrm{p}<0,05$

* $p<0,005$

t $\quad \mathrm{p}<0,01$
}

Tabla 3.

Incremento semasal de diferentes paránetros antroponétricos en ur grupo de recién nacidos de muy bajo peso alimentados con leche de su propia madre y sus controles $(\bar{x} \pm D$,E. $)$

\begin{tabular}{lccccc} 
Grupo & $\begin{array}{c}\text { Talla } \\
(\mathrm{mm} / \mathrm{sem})\end{array}$ & $\begin{array}{c}\text { Circanferencia } \\
\text { cranessa } \\
(\mathrm{mm} / \mathrm{sem})\end{array}$ & $\begin{array}{c}\text { Perínetro } \\
\text { braquial } \\
(\mathrm{mm} / \mathrm{sen})\end{array}$ & $\begin{array}{c}\text { P.T. +P.S.E. } \\
(\mathrm{mm} / \mathrm{sem})\end{array}$ & $\begin{array}{c}\text { Area magra } \\
\text { braquial } \\
(\mathrm{mm} 2 / \mathrm{sem})\end{array}$ \\
\hline L.M. & $7,4 \pm 2,4$ & $6,5 \pm 1,0$ & $1,7 \pm 0,6$ & $0,35 \pm 0,14^{*}$ & $18,2 \pm 6,3^{\mathrm{t}}$ \\
Control & $5,9 \pm 1,6$ & $5,6 \pm 1,8$ & $1,3 \pm 0,6$ & $0,17 \pm 0,20$ & $10,5 \pm 4,1$ \\
\hline
\end{tabular}

* $\mathrm{p}<0,025$

t $\mathrm{p}<0,005$ 


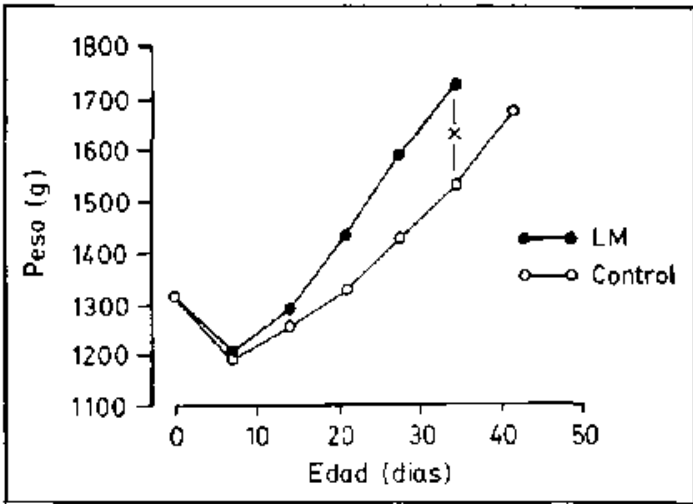

Figura 1: Curva de peso en recién nacidos de muy bajo peso de nacimiento, alimentados con leche de su propia madris (I,.M.) y su grupo control. $x p<0,05$.

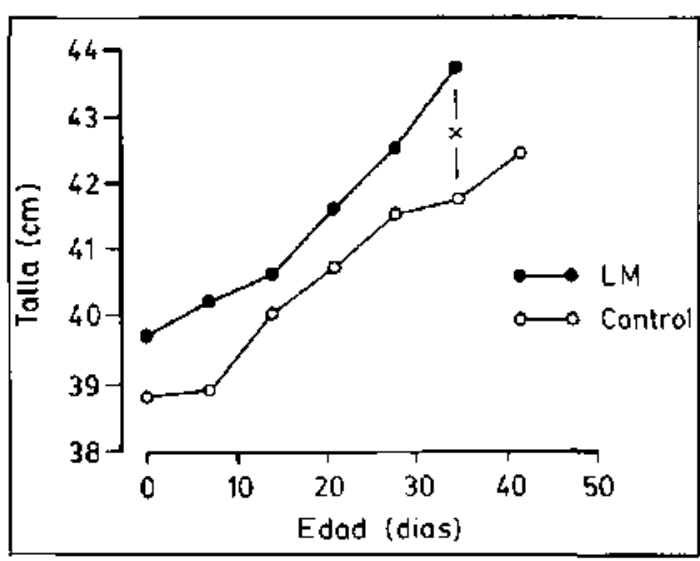

Figura 2: Curva de talla en recién nacidos de muy bajo peso de nacimiento, alimentados con leche de su propia madre (L.M.) y su grupo control, $x \mathrm{p}<0,025$.

muy bajo peso de nacimiento alimentados con leche de su propia madre crecen mejor que un grupo comparable alimentado con una fórmula láctea tnodificada de uso habitual en las unidades de recién nacidos en nuestro país. $\mathbf{E}\}$ incremento ponderal diario observado a partir de la tercera semana de vida es comparable al patrón de crecirniento intrauterino durante el último trimestre, lo que no ocurre en el grupo que recibe fórmula. Como consecuencia de ésto, el período de hospitalización es más corto y el tipo de alimentación usada permite la participación activa de la madre en la recuperación de su hijo.

En la actualidad aún no existe acuerdo con respecto a cual debería ser el pátrón de crecjmiento de los prematuros. La mayoría de los autores recomiendan como objetivo deseable la velocidad de crecimiento durante el último trimestre de vida intrauterina. ${ }^{2.16}$ Esto no es compartido por otros que postulan que el prematuro constituye una entidad diferente al feto y por lo tanto, el tratar de alcanzar este objetivo puede producir más daño que beneficios (acidosis metabólica frente a cargas grandes de proteínas, por ejemplo. ${ }^{17}$ ) A pesar de esta controversia cl crecimicnto intrauterino es el único patrón de referencia validado.

En nuestra experiencia. tras dos semanas de aparente adaptación a la vida extrauterina, los niños alimentatos con leche de su propia madre adquicren una velocidad de crecimiento comparable a la que tendrían dentro del vientre maler. no. ${ }^{14,16}$ A pesar de todos estos argumentos a su favor, la leche materna ha sido considerada por ałgunos inadecuada pata la alimentación de prematuros muy pequeños, debido a que su contenido de proteínas, sodio. calcio y fóstoro, no seria suficiente para alcanzar la velocidad de acreción de estos nutrientes observada en fetos de edad comparable. ${ }^{3}$ Estas consideraciones derivan de análisis de composición de leche producida por madres que habian dado a luz recién nacidos de término y de leches de banco, donde confluyen donantes con hijos de distintas edades gestacionales al nacer $y$ en diferentes etupas de bactancia.

Los datos derivados del análisis de lcches de madres de recién nacidos de pretérmino muestran que éstas tienen un mayor contenido de proteínas, grasa $y$ sodio $y$ una mayor densidad calórica que la descrita para leches de término. ${ }^{8-12}$ Debido a que el contenido de proteínas de la leche en las difercntes especies varía en relación directa a lá velocidad de crecimiento. expresada como el tiempo necesario para doblar el peso de nacimiento, se pensó que la leche de las madres de pretérmino pofía promover mayor crecimicnto en prema turos que la leche de banco. Este hecho ha sido demostrado por varios autores en los últimos años. ${ }^{18.19}$ Atkinsemr ${ }^{2}$ realizó estudios de balance en tres grupos de riños con características similares a los de nuestro estudio. alimentados con leche humana de banco. leche de su propia madre y fórmula láctea y demost ró gue los niños alimentados con leche de pretérmino alcanzaban retenciones nitrogenadas similares a las observadas on la vida intrauterina, con ingestas de 180 a $200 \mathrm{ml} \times \mathrm{kg} x$ día, que son similares a las administradas a los nitos del presente estudio a partir de los catorce días de vidá.

Chessex ${ }^{20}$ analizó la composición corporal de recién nacidos con peso de nacimiento inferior a $1.300 \mathrm{~g}$. alimentados con leche de su propia madre y concluyó que la composición porcentual de los diferentes nutrientes en la ganancia de peso era similar a la cormunicada pará fetos de edad gcstacional comparable.

Publicaciones más recientes 21,22 han sugerido que niños de muy bajo peso alimentados con 
lechi fresca de su propia madre tendría un crecimiento inferior al observado con el uso de fórmulas lácteas especialmente discinađas para prematuros, considerando la acreción intrauterina de los diferentes nutrientes. Sin embargo, dcbido a que en nuestro medio no contamos con este tipo de formulas, nos parece que los esfuer. zos deben ir dirigidos a promover la alimentación del prematuro con leche de su propia madre.

Es importante considerar que el contenido de calcio y fósforo de la leche de pretérmino está aparentemente por debajo del requerido para obtener una acreción de estos nutrientes. similar a la observada en la vida intrauterina ${ }^{23}$ y yus, por lo tanto, para evitar la enfermedad osea metabólica seria necesarjo administrar un suplemento diario de arnbos mincrales. de acuerdo a lits acluales recomendaciones. ${ }^{2,16}$

Además de sus proyecciones nutricionales, el uso de leche materna en la alimentación del promaturo mantiene a la madre más cercana a su hijo durante la hospitalización, la hace sentirse partícipe en su recuperación y contribuye de esta manera al establecimiento de una mejor relación madre-hijo, hecho de vital importancia si se considera que la separación de ambos tiene lugar en ur momento crítico para el cstablecimiente de] vículo afectivo, y promueve la mantención de la lactancia materna más allá del alta del nifio con todas las ventajas que esto implica y que ban sido discutidas ampliamente en la literatura. 1,14

Cabe destacar que la menor duración de la hospitalización debido a que alcanzan más rápidamente los $2.000 \mathrm{~g}$ de peso, reduce los dias-incubadora y días-cuna. 10 que contribuye a disminuir el costo recuperacional de estos niños y, por lo tanto implica un beneficio económico.

\section{RESUMEN}

Con el objeto de evaluar el crecimiento de recien nacidos menores de $1.500 \mathrm{~g}$ alimentados con leche de su propia madre (L.M.) se estudiaron 10 niños con peso promedio de $1.315 \pm$ $128 \mathrm{~g}$ y edad gestacional de $31,1 \pm 1,5$ semanas, adecuados para la edad gestacional y sin malformaciones congénitas mayores y se compararon con un grupo control de caracter ísticas similares alimentado con fórmula artificial (NAN ${ }^{\circledR} 13 \%$ ). La pérdida inicial de peso fue de $10,9 \%$ para el grupo L.M. y de $9.8 \%$ para el control ( $p=$ N.S.). La recuperación del peso de nacimiento ocurrió a los 16,0 dias en el grupo L.M. y a los 20,8 en et control $(p<0,05)$. La ganancia ponderal posterior fue de $23,7 \mathrm{~g} \times$ día versus 17,5 en el control $(p<0,005)$. El tiempo empleado en alcanzar los $2.000 \mathrm{~g}$ fue de $46 \pm 10$ días en el grupo L.M. $y$ de $63 \pm 17$ en el control $(p<0,01)$. En recién nacidos de muy bajo peso la leche de su propia madre promueve crecimiento ponderal mejor que la fórmula de uso habitual: disminuye el período de hospitalización y favorece la relación madre-hijo.

\section{REFERENCIAS}

1. Amorican Academy of Pediarrics: Bueast Feeding A Commentary in Celebration of the lnternational Ycar of the Child, 1979. Pediatrics 62. 591, 1978 .

2. Commistee on Nutrition American Academy of Pediatrics: Nutricional Needs of Low Birth-Weight Intants. Pediatrics 7S:976, 1985.

3. Fomon, S.J. Ziegler, E.F., Vásque'z, H.D.: Human Milk and the snall premature infant Am J Dis Child 131: 473. 1977.

4. Forbes, G,B.: Human milk and the small baby. Am $J$ Dis Child 136: $577,1982$.

5. Gerdon, f.H, Levine, S.Y., and Mc Namara, H.: Feeding of the premature infant. A comparison of human and cow's milk. Am J Dis Child 73: 442, 1947.

6. Dovies, D.P.: Adequacy ol expressed bredst milk for early growth of preterm infants. Arch Dis Child 52: $296,1977$.

7. Tyson. J.E. Lasky, R.E., Mize, C.E., Richards, C.I., Btair-Smith, $H_{+}$Whyte, $R$, and Bear, A.E.: Growth, metabolic response and development in very low-birth-weight infants fed banked human milk or enriched formula Veonatal findings. J Pediatr 103: 95, 1983 .

8. Gross. S.J., Datid, R.J., Batman, L. and Tomarelli, R.M.: Nutritional composition of milk produced by mothers delivering preterm. J Pediatr 96: 641, 1980 .

9. Sann, L., Bienvent, F. Lahet, C. Bienvenu, J., Bethenod, $M$.: Comparison of the composition of breast milk from mothers of term and preterm jnfants Acta Pediatr Scand 70: 115, 1981.

10. Anderson, G.H., Atkinson, S.A. and Bryan, M.H.: Energy and macronutrient content of human milk during early lactation from mothers giving birth prematurely and at term. Am J Clin Nutr 34: 258, 1981

11. Arkinson, S.A., Bryan, M.H., and Anderson, $G, H$.: Human milk differences in nitrogen concentration in milk ftom mothers of term and premature infants. J Pediatr 93: 67, 1978.

12. Atkinson, G.H.: Human milk feeding. Pediatr Clin North Am 32: 335, 1985.

13. Dubowitz, L.M.S., Dubowitz, V.. Goldberg. C.: Clinical assessment of gestational age in the newborn infant. J Pediatr $77: 1,1970$.

14. Lubchenco, L., Honsman, $C$. Dressler, $M$, et ol: Intrauterine growth as estimated from liveborn birth weight data at 24 to 42 weeks of gestation. Pediatrics 32: 793, 1963.

15. Firisancho, A.R.: Triceps skinfold and upper arm rnuscle size norms for assessment of nutritional status. Am J Clin Nuts 27: 1052, 1974.

16. Ziegler, E.E., Biga, R.L., Fomon. S.J.: Xutritional requirements for the premature infant, in Suskind, R.M. (ed): Textbook of Pediatric Nutrition. New York, Raven Press: 29, 1981.

17. Raiha, N.C.R., Heinonen. K., Rassin, D.K., Gault, G.E. Milk protein quantity and quality in lowbirthweight infants: I Metabolic responses and effect on growth. Pedia trics 57: 659, 1976. 
18. Gross, S.J.: Growtl and biochemical response of preterm infants led human milk or modifjed intant tormula. N Fingl J Med 308: 237, 1983.

19. A skinson, S.A., Brvan, M.H. and Anderson, G.H.: Human milk feeding in premature infants. Protein. fat and carbohydrate balances in the first two weeks of lite. J Pediatr 99: 617, 1981.

20. Chesses, P., Reichman, B., Verellen, $G$., Putet, G., Smirh, J.M, Heim, T. and Sw'ver, P.R.: Quality of growtly in premature infants fed their ow mothers milk. J Pediatr 102: 107. 1983.

21. Hendrickse, WA, Spencer, S.A., Roberton, D.M. and Hull, D.: The caloric intake and weight gain of low bit th weight infants fed on fresh breast railk or a special formula milk. Eur J Pediatr 143: 49, 1984.

22. Schandler, R.J., Garza, C. and Nichols. B.L.: Fortified mothers' milk for very low birth weight infants: Results of growth and nutrient balance studies. J Pediatr 107; 437, 1985.

23. Atkirson, S.A., Radde, J.C., Anderson, G.H.. Matromincral balances in ptemature infants fed their own mothers' milk or formula. J Pediatr 102: 99, 1983.

24. Lemons. P., Stuart, M., Lemons, J.A.: BreastFeeding the Premature Infant Clin Perinat 13: 111, 1986 . 